\section{MOTIVES FOR CHOOSING FOOD: A COMPARISON BETWEEN YOUNG ADULTS RESIDING IN UK AND SINGAPORE}

L. Santos-Merx, H. Kendall, H. Ong, K. Westerman. Newcastle University Singapore, $172 A$ Ang Mo Kio Ave 8, 567739, Singapore

Eating out and consuming ready meals is popular among young people in developed countries. Such habits are often associated with large portions, excessive fat/sodium with possible adverse health consequences. Understanding the drive for choosing food has been crucial for product development but should also be considered in public health interventions. Hence, the aim of this study is to contrast food choice motivations of young adults residing in UK and Singapore by applying Steptoe's Food Choice Questionnaire (FCQ). Gender and nutrition knowledge were considered as additional influences on food choice motivations. The sample included 234 adults from UK ( $n=115,45 \%$ females) and Singapore ( $n=119,59 \%$ females) aged 18-28 years. Mann-Whitney analyses of the FCQ subscales mean scores showed Singaporean participants (all and females) to give more importance to Mood, Convenience, Natural Content, Familiarity and Ethical Concern than UK participants. Likewise Singaporean male participants favoured the previous motives with the exception of Natural Content. UK and Singaporean participants, regardless of gender, equally rated Health, Sensory Appeal, Price and Weight Control. No significant differences between participants with and without nutrition knowledge were found. However, females with nutrition knowledge gave more importance to Natural Content and Familiarity than males. Interestingly, males without nutrition knowledge gave more importance to Health than females. In this small comparative study, several motives for food choice were surprisingly similar in young adults from two culturally divergent countries. Moreover, nutrition knowledge and gender appear not to be strong influences underlying food choices among these young adults.

http://dx.doi.org/10.1016/j.appet.2017.11.056

\section{ACUTE COGNITIVE AND BLOOD GLUCOSE EFFECTS OF BLUEBERRY ANTHOCYANINS IN HEALTHY YOUNG ADULTS}

L. Bell, D.J. Lamport, L.T. Butler, C.M. Williams. School of Psychology and Clinical Language Sciences, University of Reading, RG6 6AL, UK

A study by Rodriguez-Mateos et al (2014) reported acute, dose-dependent vasodilatory effects following consumption of blueberry anthocyanins. Increased cerebral blood flow resulting from vasodilation induced by blueberry, or any similar flavonoid-rich intervention, has been posited as a mechanism for acute improvements in cognition. A study by Torronen et al (2009) reported that berry anthocyanins also confer glucoregulatory benefits in the immediate postprandial period. Improved glucoregulation may provide an additional mechanism for improved cognition. This double-blind, crossover study investigated the acute effects of three separate wild blueberry doses and a sugar-matched control on cognition and postprandial glucose outcomes in 41 healthy young adults. Drinks, containing $0,310,517$, and $724 \mathrm{mg}$ blueberry anthocyanins, matched to the Rodriguez-Mateos et al. (2014) vascular study, were administered over four test days, each separated by a one week washout period. Cognition and mood measures including: affect (PANAS), mental fatigue (Likert questionnaire), attention (digit vigilance and RVIP) and working memory (serial subtraction), were assessed pre-, 1 hour, and 2 hours post-consumption of each dose. Blood glucose concentrations were measured immediately prior to cognitive testing at each time point and at additional 15 and 30 minute postprandial time points in order to determine a glucose response profile. A dose-dependent attenuation of glucose peak was observed for the blueberry conditions when compared with the control. No significant cognitive effects were observed for the PANAS, mental fatigue or digit vigilance measures. Data analysis is ongoing for other measures. However, preliminary results suggest that while blueberry anthocyanins confer physiological benefits, these do not translate into cognitive improvements for a young adult population.

\section{EFFECT OF ORO-SENSORY EXPOSURE ON CEPHALIC PHASE RESPONSES AND SATIATION}

M. Lasschuijt, M. Mars, M. Stieger, S. Miquel-Kergoat, C. de Graaf, P. Smeets. Division of Human Nutrition, Wageningen University \& Research, Stippeneng 4, 6708 WE Wageningen, The Netherlands

Oro-sensory exposure (OSE) to food helps to regulate food intake by inducing cephalic phase responses, which may induce an early onset of satiation. However, the effects of the duration and intensity of OSE on cephalic phase responses and satiation are unknown.

The objective was to determine the effect of variation in OSE duration and intensity on endocrine and metabolic cephalic phase responses and satiation. Increasing OSE duration and intensity was hypothesized to increase the magnitude of cephalic phase responses (insulin, ghrelin, PP, glucose, FFA) and lower food intake. Eighteen males $(22.3 \pm 2.5 \mathrm{y}$, BMI $22.4 \pm 1.8 \mathrm{~kg}$ $\mathrm{m}^{2}$ ) participated in a $2 \times 2$ randomized cross-over design with control condition (no MSF) in which they modified sham fed four gel-based model foods while blood samples were collected at 8 time points. Model foods differed in chewing duration (hard or soft texture) and sweetness (low or high intensity). They were matched on caloric content, flavour and palatability. After MSF subjects received an ad libitum lunch meal (bread with sweet and savoury toppings). Preliminary partial analyses showed no effect of texture and sweetness on glucose levels at any of the time points. In addition, we found no effect of texture $(\mathrm{F}=0.13, \mathrm{P}=0.72)$ and sweetness $(\mathrm{F}=0.30, \mathrm{P}=0.59)$ on total intake during the ad libitum lunch. No difference in intake of the savoury or sweet toppings between texture and taste levels was found. These results indicate that OSE by modified sham feeding does not induce a glucose CPR or decrease in subsequent food intake. Analysis of the remaining endocrine and metabolic outcome measures is still in progress.

http://dx.doi.org/10.1016/j.appet.2017.11.058

\section{DOWNSIZING PORTIONS OF HIGH ENERGY-DENSE SNACK FOOD AND SUGAR-SWEETENED BEVERAGES IN ADOLESCENTS: THE SMART SNACKING STUDY}

M. Sharps, C. Evans, M. Hetherington. School of Food Science and Nutrition, University of Leeds, Woodhouse Lane, Leeds, LS2 9JT, UK

In the UK in $201324 \%$ of boys and $23 \%$ of girls were classed as overweight or obese (Ng, et al., 2014). Increasing portion sizes are believed to contribute to the obesity problem (Rolls, Engell, \& Birch, 2000), with people eating more when exposed to larger portions (Fisher, Liu, Birch, \& Rolls, 2007). Portion sizes of high energy-dense food and drinks have been shown to be associated with higher energy intake and BMI in adolescents (Albar, Alwan, Evans, \& Cade, 2014). Therefore, targeting adolescents' portion sizes is crucial. Since adolescents' eating behaviour has been shown to be influenced by the eating behaviour of their peers (Romero et al., 2009), peer-led nudging may be a way of encouraging adolescents to reduce their portions. The aim of the Smart Snacking study is to examine whether peer-led nudging using social media may be a way of reducing portion sizes in adolescents. We plan to run a feasibility study to identify the typical portion sizes of adolescents, and an intervention using social media which aims to encourage adolescents to downsize their portions of high energy dense snack foods and sugar-sweetened beverages. We will discuss the results of the feasibility study and the development of the intervention.

http://dx.doi.org/10.1016/j.appet.2017.11.059 